Regards sur l'économie allemande

Bulletin économique du CIRAC

$65 \mid 2004$

Varia

\title{
La difficile émergence d'un marché de la lettre en RFA
}

Isabelle Bourgeois

\section{CpenEdition}

Édition électronique

URL : http://journals.openedition.org/rea/3942

DOI : $10.4000 /$ rea.3942

ISBN : 978-2-8218-0826-3

ISSN : 1965-0787

\section{Éditeur}

CIRAC

\section{Édition imprimée}

Date de publication : 1 mars 2004

Pagination : 23-28

ISSN : 1156-8992

\section{Référence électronique}

Isabelle Bourgeois, "La difficile émergence d'un marché de la lettre en RFA », Regards sur l'économie allemande [En ligne], 65 I mars 2004, mis en ligne le 29 octobre 2009, consulté le 30 avril 2019. URL : http://journals.openedition.org/rea/3942 ; DOI : 10.4000/rea.3942 


\section{La difficile émergence d'un marché de la lettre en RFA}

\section{Isabelle Bourgeois}

Dès 1998, en application de la Directive européenne 97/67/CE, l'Allemagne avait ouvert à la concurrence son marché postal et engagé la privatisation de l'opérateur historique. Deutsche Post AG s'était vu attribuer une licence légale exclusive maintenant son monopole sur le marché de la lettre d'ici la libéralisation totale du marché, prévue initialement en 2003, puis repoussée au $1^{\text {er }}$ janvier 2007. Ce "secteur réservé ", qui correspond au cœur de la mission de "service universel ", concerne aujourd'hui les lettres de moins de $100 \mathrm{~g}$. Le segment des lettres de 100 à 1000 g, quoique ouvert à la concurrence, est soumis au régime des licences jusqu'à fin 2006. Cette réglementation transitoire, semblable à celle des télécommunications, est destinée à ménager la transition vers l'achèvement du marché des services postaux.

Bien que sa durée soit réduite de deux ans par rapport aux délais prévus par la nouvelle Directive postale 2002/39/CE, la prolongation du monopole de Deutsche Post AG dans le segment de la lettre s'attire les plus vives critiques à la fois de l'autorité de régulation RegTP et de la Commission des Monopoles. Elle se traduit en effet par une augmentation considérable du ticket d'entrée sur le marché des activités soumises à licence, l'opérateur historique multipliant les procédures à l'encontre de ses concurrents. Dès lors, les gardiens de la concurrence plaident pour une levée anticipée de la licence légale exclusive ou, à défaut, pour une réduction drastique de son périmètre.

Le volume de l'ensemble du marché postal allemand - lettre, colis, express et logistique confondus - représente un chiffre d'affaires de plus de 23 milliards $€$ en 2003, selon la RegTP ; Deutsche Post AG (DPAG) en réalise les deux tiers. Ce marché se caractérise par le fait que le segment du colis, de l'express et de la logistique, soit une bonne moitié du total, est privé depuis longtemps. L'autre segment, celui de la lettre de moins de $1000 \mathrm{~g}$, qui représente quelque $10 \mathrm{mil}-$ liards $€$, est actuellement en voie de libéralisation. DPAG y tient encore $96 \%$ du marché. Six ans après l'ouverture de ce marché, les nouveaux opérateurs n'en ont donc conquis que $4 \%$. Autrement dit : l'opérateur historique reste en position de monopole de fait sur l'ensemble du marché postal.

Parts de marché dans le segment des activités postales soumises à licence (lettre)

\begin{tabular}{|l|r|r|r|r|}
\cline { 2 - 5 } \multicolumn{1}{c|}{} & $\mathbf{2 0 0 0}$ & $\mathbf{2 0 0 1}$ & $\mathbf{2 0 0 2}$ & $\mathbf{2 0 0 3}^{*}$ ) \\
\hline Total du marché (en mio $€$ ) & 10300 & 10200 & 10200 & 10100 \\
\hline CA des opérateurs (hors DP AG ; en mio $€$ ) & 173,7 & 248,8 & 305,5 & 400,0 \\
\hline PDM des opérateurs licenciés (en \%) & 1,7 & 2,4 & 3,0 & 4,0 \\
\hline PDM de DP AG (en \%) & 98,3 & 97,6 & 97,0 & 96,0 \\
\hline CA des « services de qualité supérieure » (en mio $€$ ) & 46,5 & 83,0 & 125,8 & 180,0 \\
\hline PDM des « services de qualité supérieure » (en \%) & 0,45 & 0,81 & 1,2 & 1,8 \\
\hline
\end{tabular}

Source des données : RegTP, Rapport d'activité 2002/2003, décembre 2003. *) Estimations.

L'autorité de régulation Regulierungsbehörde für Post und Telekommunikation (RegTP) a recensé 896 détenteurs de licences au 30-09-2002. II s'agit surtout (799) de licences attribuées depuis 1998, les autres ayant été accordées en
Deutsche Post AG en monopole de fait
Les détenteurs de licences sont essentiellement des PME 
1995, après l'entrée en vigueur de la loi Postreform I/ qui amorçait la privatisation partielle de la poste allemande. Ces nouveaux acteurs sont principalement des petites entreprises réalisant un CA annuel de moins de $500000 €$. Seulement 80 d'entre eux étaient des entreprises de taille moyenne. Dans la mesure où le marché de masse est encore réservé à DPAG, ces opérateurs doivent en effet se contenter d'activités de niche. Et ces activités ne sont encore guère rentables: sur les 565 entreprises ayant communiqué leur bilan à la RegTP, 229 seulement étaient excédentaires en 2002 ; 108 étaient déficitaires.

\section{Le marché postal allemand est de jure ouvert à la concurrence depuis 1998}

Le marché postal allemand est ouvert depuis le 01-01-1998, date d'entrée en vigueur de la Loi Postgesetz du 22-12-97 transcrivant en droit national la Directive postale 97/67/CE. Depuis, le principe constitutionnel de la liberté d'entreprise (art.12 de la Loi fondamentale) et, partant, le droit commun de la concurrence, s'appliquent à tous les segments et types de services postaux, sauf deux, provisoirement soumis à un régime particulier.

Initialement, le marché postal allemand devait être ouvert totalement le $1^{\text {er }}$ janvier 2003 . Mais l'adoption, en mai 2002, de la Directive 2002/39/CE qui reporte à 2009, voire au-delà, " l'achèvement du marché intérieur des services postaux », a amené l'Allemagne a reporter l'ouverture de son marché postal à janvier 2008. La transcription de la directive en droit national s'est traduite également par une modification du périmètre du " secteur réservé » et l'obligation faite au seul opérateur historique de remplir des missions de " service universel » en contrepartie du prolongement de sa licence légale exclusive.

Le marché postal allemand est donc libre, à deux exceptions près, adoptées à titre transitoire jusqu'à fin 2007 :

- le « secteur réservé ». Le segment des lettres de moins de $100 \mathrm{~g}$, affranchies à un tarif inférieur ou égal à $1,35 €$ (soit trois fois le tarif de base), fait l'objet d'une licence légale exclusive attribuée à Deutsche Post AG. Ces seuils sont ceux de la Directive 2002/39/CE et ont été introduits le 01-01-2003 ; ils étaient auparavant de $200 \mathrm{~g}$ et cinq fois le tarif de base. Au $1^{\text {er }}$ janvier 2006, ils seront de nouveau abaissés, passant à $50 \mathrm{~g}$ et un tarif de deux fois et demie au maximum celui de la lettre standard;

- le « secteur soumis à licence». Le segment des envois d'un poids inférieur ou égal à $1000 \mathrm{~g}$ (hors " secteur réservé ») est ouvert à la concurrence, mais les opérateurs sont soumis à licence. Ces licences sont délivrées sur simple demande par l'autorité de régulation des postes et télécommunications, la Regulierungsbehörde für Post und Telekommunikation (RegTP). Elles ne peuvent être refusées aux candidats que pour des motifs graves comme l'absence patente de compétence professionnelle ou un risque pour la sécurité publique.

Depuis le 01-01-2003, ces licences sont classées en huit catégories, déclinées selon divers échelons territoriaux :

A : lettres et catalogues de plus de $100 \mathrm{~g}$ et affranchis respectivement à au moins trois fois le tarif de la carte postale ou du catalogue de moins de $20 \mathrm{~g}$;

B : petit courrier de publipostage (50 exemplaires minimum, d'un poids supérieur à $50 \mathrm{~g}$ );

C : courrier inter-entreprises (l'expéditeur dépose le courrier chez le prestataire, où le destinataire va le chercher, les trois partenaires étant liés par un contrat de services) ;

D : services de "qualité supérieure » se différenciant des prestations de service universel par leur valeur ajoutée ;

E : collecte d'envois postaux chez l'expéditeur et acheminement vers un guichet de DP AG ;

$\mathbf{F}$ : collecte des envois dans les boîtes postales de DP AG et distribution aux destinataires ;

G : courrier transfrontière sortant ;

$\mathbf{H}$ : acheminement du courrier transfrontière entrant vers une plateforme de DP AG.

Une grande fluctuation sur le segment des licences
Entre 1998 et la fin octobre 2003, la RegTP a attribué des licences à 1460 entreprises au total et n'a été amenée à en retirer que cinq. Mais le nombre d'entreprises en activité est inférieur, étant donné qu'un tiers des détenteurs de licences (466) s'est retiré du marché. Si quelques entreprises ont déposé leur bilan (35) ou fermé pour d'autres raisons (70), elles sont tout de même 356 à avoir rendu leur licence. Cette fluctuation est le propre de tout marché en cours de libéralisation, où la seule perspective d'ouverture attire en masse de nouveaux entrants ; ensuite, le nombre des acteurs se réduit pour finir par se consolider. Dans le cas des licences postales s'y ajoute le fait que, depuis le 4 février 2002, date d'entrée en vigueur du décret Post-Lizenzgebührenverordnung, l'octroi d'une licence est soumis à redevance (comprise entre 175 et $700 €$ ). La RegTP avait alors offert aux entreprises licenciées avant cette date la possibilité de rendre leur licence sans frais ; 105 d'entre elles ont saisi l'opportunité pour se retirer d'un marché à haut risque commercial et, plus encore, procédurier. 
CA des détenteurs de licence par catégorie (en mio $€$ )

\begin{tabular}{|c|c|c|c|c|}
\hline Licences & 2000 & 2001 & 2002 & $\left.2003^{\star}\right)$ \\
\hline $\mathrm{A}\left(\right.$ ancien périmètre ${ }^{\mathrm{a}}$ ) & 32,9 & 37,3 & 40,6 & - \\
\hline$A$ (nouveau périmètre ${ }^{b}$ ) & - & - & - & 57 \\
\hline B (petit publipostage) & 60,2 & 86,4 & 92,5 & 100 \\
\hline C (courrier inter-entreprises) & 0,5 & 0,5 & 1,2 & 1 \\
\hline $\mathrm{D}$ (services « de qualité supérieure ») & 46,5 & 82,9 & 125,8 & 180 \\
\hline E (acheminement vers un guichet de DP AG) & 4,0 & 4,8 & 9,2 & 9 \\
\hline $\mathrm{F}$ (collecte dans les boîtes postales de DP AG) & 2,9 & 3,7 & 3,6 & 4 \\
\hline G (courrier transfrontière sortant) & - & - & - & 15 \\
\hline $\mathrm{H}$ (courrier transfrontière entrant) & - & - & - & n.c. \\
\hline Licences antérieures (envois en masse ${ }^{c}$ ) & 26,7 & 33,0 & 32,6 & 34 \\
\hline Total & 173,7 & 248,6 & 305,5 & 400 \\
\hline
\end{tabular}

Source des données : RegTP, Rapport d'activité 2002/2003, décembre 2003. *) Estimations. a) jusqu'au 31-12-02 : poids $>200 \mathrm{~g}$ ou prix $>2,55 € ;$ b) depuis le 01-01-03 : respectivement $>100 \mathrm{~g}$ et $>1,35 € ;$ c) attribuées entre le 01-01-95 et le 01-01-98.

Les licences les plus prisées sont de loin celles des catégories $E, F$ et $D$. Ce sont celles qui permettent d'offrir des services à forte valeur ajoutée : le CA des seuls services " de qualité supérieure » (licence $D$ ) a presque quadruplé depuis 2000. A l'inverse, la part des licences portant sur les activités de transport et d'acheminement du courrier (licences antérieures à 1998 et licence B, notamment) est en recul. La prorogation de la licence légale exclusive de Deutsche Post AG contribue à inciter les concurrents potentiels à différer leurs investissements sur ce marché de masse particulièrement intensif en main d'œuvre.

Répartition territoriale des détenteurs de licences au 30-09-2003

\begin{tabular}{|l|r|rrrrrrrr|}
\cline { 2 - 9 } \multicolumn{1}{c|}{} & Total & A & B & C & D & E & F & G & H \\
\hline Bade-Wurtemberg & 122 & 84 & 82 & 47 & 84 & 107 & 96 & 5 & 5 \\
\hline Basse-Saxe & 165 & 108 & 105 & 61 & 142 & 138 & 129 & 4 & 4 \\
\hline Bavière & 92 & 55 & 51 & 35 & 59 & 80 & 80 & 3 & 3 \\
\hline Berlin & 46 & 28 & 27 & 19 & 33 & 41 & 37 & 2 & 2 \\
\hline Brandebourg & 77 & 41 & 54 & 29 & 67 & 64 & 61 & 2 & 2 \\
\hline Brême & 5 & 5 & 5 & 4 & 4 & 4 & 3 & 0 & 0 \\
\hline Hambourg & 37 & 23 & 20 & 6 & 11 & 32 & 33 & 3 & 3 \\
\hline Hesse & 71 & 49 & 45 & 28 & 53 & 62 & 57 & 7 & 6 \\
\hline Mecklembourg-Pom. & 62 & 39 & 36 & 22 & 60 & 50 & 47 & 4 & 4 \\
\hline Rhénanie du N.-W. & 332 & 214 & 211 & 144 & 262 & 281 & 274 & 25 & 23 \\
\hline Rhénanie-Palatinat & 60 & 46 & 45 & 34 & 52 & 56 & 55 & 2 & 2 \\
\hline Sarre & 13 & 9 & 11 & 9 & 9 & 11 & 11 & 1 & 1 \\
\hline Saxe & 146 & 99 & 99 & 68 & 133 & 116 & 109 & 25 & 25 \\
\hline Saxe-Anhalt & 87 & 61 & 55 & 40 & 79 & 76 & 76 & 3 & 3 \\
\hline Schleswig-Holstein & 60 & 52 & 48 & 33 & 50 & 51 & 50 & 3 & 3 \\
\hline Thuringe & 69 & 34 & 46 & 21 & 65 & 58 & 55 & 0 & 0 \\
\hline UE *) & 2 & 2 & 2 & 2 & 1 & 2 & 2 & 0 & 0 \\
\hline Total & 1446 & 949 & 942 & 602 & 1164 & 1231 & 1175 & 89 & 86 \\
\hline
\end{tabular}

Source des données : RegTP, Rapport d'activité 2002/2003, déc. 2003. *) Détenteurs ayant leur siège dans un Etat membre de l'UE. NB : le total est supérieur aux 896 détenteurs recensés par la RegTP, nombre d'entre eux disposant de plusieurs licences.

Au total, les entreprises sous licence ont créé plus de 23000 emplois en 2002. La RegTP insiste sur le fait qu'il s'agit de créations et non d'un transfert d'emplois de DPAG, qui avait supprimé 23000 emplois à temps plein dans le cadre de sa rationalisation. Dans le secteur soumis à licence, la majorité des emplois (14 200) sont des " petits boulots » : essentiellement des « mini-jobs » (moins de $325 €$ par mois et moins de 15 heures travaillées par semaine) ; le travail occasionnel ou saisonnier (revenus non soumis à cotisations sociales) ne représente que 3113 emplois au total. Ils ont été surtout créés dans des régions en restructuration, principalement dans les nouveaux Länder (8 363 emplois, dont

Les services à valeur ajoutée sont les plus attractifs
Les PME sous licence ont créé 23000 emplois 
Un marché à fort potentiel, mais avec de fortes entraves

près de la moitié dans le seul Mecklembourg). Les activités postales leur sont un outil parmi d'autres de reconversion industrielle.

Le potentiel économique de la libéralisation du marché postal est énorme, aux dires de la Commission des Monopoles, qui tire le parallèle avec l'impact de l'ouverture du marché des télécommunications dans un récent rapport sur l'état de la concurrence sur ces deux marchés. Mais alors que cette commission indépendante chargée d'évaluer l'état de la concurrence et de formuler des propositions de réforme du droit en la matière, salue " l'intensification de la concurrence » dans les télécommunications, elle critique vertement " un monopole postal coulé dans le ciment » entravant la constitution d'un marché de la lettre jusque dans les segments de niche. La réduction du périmètre de la licence légale exclusive aux envois de moins de $50 \mathrm{~g}$ en 2006 n'y changera rien car, même alors, "quelque $60 \%$ du marché de la lettre resteront soumis à monopole ». La principale cause en est la licence légale exclusive de DPAG, d'autant qu'elle favorise la pratique des « subventions croisées », DPAG finançant son expansion dans le colis et la logistique grâce aux recettes issues du secteur réservé. Cela constitue une entrave à la concurrence au regard du droit national comme du droit communautaire, mais seule la Commission européenne intervient, alors que la RegTP reste singulièrement muette, critique la Commission des Monopoles. Pourtant, « un contrôle strict des abus de position dominante est la condition sine qua non de l'émergence d'un marché ».

\section{Licence légale exclusive : un conflit entre droit constitutionnel et droit commun}

La Commission des Monopoles s'insurge contre le Tribunal constitutionnel fédéral qui, dans un arrêt du 7 octobre 2003, avait déclaré conforme à la Loi fondamentale la prolongation de la durée de la licence légale exclusive. II avait débouté la plainte de six détenteurs de licences postales qui estimaient que cette prolongation s'assimilait à une violation du principe de la liberté d'entreprise (art. 12 de la Loi fondamentale). Ils estimaient en outre que, contrairement à l'objectif affiché (garantie de l'intérêt général), cette disposition était destinée en réalité à soutenir le cours de l'action de Deutsche Post AG et à protéger l'opérateur contre la concurrence.

La Cour avait rejeté la plainte quant au fond (violation de l'art. 12) au motif que le législateur avait exercé légitimement son droit de limiter, à titre transitoire et lorsque l'intérêt général le justifie - mais seulement dans ce cas -, l'application du principe fondamental de la liberté d'entreprise. En l'occurrence, il s'agissait, pour assurer la poursuite du service universel, « d'éviter un changement systémique brutal » et de "garantir que la libéralisation nationale se poursuit en harmonie avec l'évolution européenne »; or la libéralisation du marché postal européen « n'est dans l'ensemble pas réalisée », rappellent les juges de Karlsruhe.

Dans la lecture de la Commission des Monopoles, les missions de service universel et la « réciprocité de l'ouverture des marchés en Europe », loin de ménager la transition en douceur vers le marché, remettent bien plutôt « en question le principe même de la libéralisation des marchés de la lettre, vidant de sa substance la modification de la Loi fondamentale en 1994 ». Cette modification avait été nécessaire pour désétatiser les services postaux avant même de les libéraliser (voir REA 47/00) et pour procéder à la séparation entre pouvoirs régaliens de l'Etat, structure publique et missions d'intérêt général. Enfin, précise la Commission, le principe d'un monopole limité dans le temps n'avait été adopté qu'aux fins exprès de permettre à l'opérateur historique d'apurer le passif des retraites. Or ce dossier est clos depuis 2000. Economiquement, plus rien ne justifie donc son monopole, même si le droit constitutionnel permet de le proroger un temps.

Un ticket d'entrée élevé : la TVA dont est exonérée DP AG

La marque « Post ${ }^{\circledR}$ » entrave les concurrents
Vu sous l'angle macro-économique, l'inactivité de la RegTP est avérée. Et aussi bien la Commission des monopoles que les fédérations des opérateurs postaux font en outre grief à l'autorité de régulation d'avoir favorisé DPAG en fixant à un niveau trop élevé le tarif d'affranchissement de la lettre. Par ailleurs, elles s'insurgent contre le législateur qui, au nom du service universel, dispense de TVA les services de DPAG dans son domaine réservé. La conséquence en est que ses concurrents, non exonérés, doivent proposer leurs services à des tarifs inférieurs d'au moins le montant de la TVA, soit $16 \%$. Cela réduit d'autant leurs marges et ralentit donc l'émergence d'un secteur concurrentiel. Contraints ainsi de comprimer leurs coûts, et surtout leur masse salariale, ils recourent massivement aux « petits boulots ».

Mais la RegTP est amenée à concentrer son énergie sur la lutte contre une entrave à la concurrence qui, sans être prise en compte par la loi du même nom, 
se révèle le principal frein à l'essor d'un marché concurrentiel de la lettre : le risque procédurier systématique que fait peser DPAG sur ses concurrents. II prend essentiellement deux aspects. Le premier, de loin le moins pesant : à l'instar des précédents dans le transport ferroviaire («Die Bahn ® ») et les télécommunications («Telekom $® »)$ l'opérateur historique a fait enregistrer en novembre dernier auprès de l'Office allemand des Brevets et des Marques la marque« Post $\circledast$ ». L'appellation est donc protégée depuis le dépôt de la demande d'enregistrement en février 2000, ce qui permet à l'opérateur de recourir en justice contre ses concurrents. Divers contentieux existent, dont un avec la société EP Europost, un joint venture entre la Poste néerlandaise et Hermes Logistik, une filiale du vépéciste Otto. La Fédération des Services internationaux d'express et de courrier (KIEP) vient de répliquer en janvier en déposant une demande d'annulation, estimant que le terme de " poste » est depuis toujours un générique. L'affaire est susceptible de remonter jusqu'à la Cour fédérale de Cassation.

Le second risque procédurier est de loin le plus lourd. II affecte essentiellement les détenteurs d'une licence $D$ (services de qualité supérieure), soumis à un tir nourri de contentieux administratifs et civils. Dès la première année de l'ouverture du segment soumis à licences, la RegTP recensait quelque 300 procès intentés par DPAG. Certes, conformément à son rôle de défenseur des concurrents de l'opérateur historique, la RegTP est parvenue l'an dernier à transférer la plupart des contentieux devant les tribunaux administratifs, ce qui réduit au moins les frais de procédure pour les nouveaux entrants. Mais ce n'est qu'un expédient tant que la juridiction suprême ne lèvera pas l'incertitude juridique sur les contours précis des services de qualité supérieure, permettant alors aux entreprises de planifier leurs activités en toute sécurité.

\section{Licence D : quelles prestations pour des « services de qualité supérieure 》?}

Les critères relatifs aux prestations de la Licence $D$, tels que les énumère la Loi Postgesetz de 1997 amendée en 2002, sont les suivants : ces prestations doivent être différentes des services liés au service universel, elles doivent présenter des caractéristiques particulières et elles doivent être de qualité supérieure. Cette définition étant source de litige car trop générale, la RegTP en a précisé les contours dès 1999. Dans sa définition, sont dits de qualité supérieure les services remplissant les cinq critères ci-dessous :

1 - prise en charge des lettres chez le client, à des heures fixes ou sur rendez-vous ;

2 - acheminement de la lettre au destinataire le jour même de la prise en charge et/ou si la prise en charge s'effectue après 17 heures, livraison au destinataire avant 12 heures le lendemain s'il s'agit d'un jour ouvrable et/ou à la date fixée par le client :

3 - facturation périodique a posteriori ;

4 - possibilité de rerouter la lettre pendant l'acheminement ;

5 - garantie contractuelle de non facturation de la prestation en cas de non respect des délais par le prestataire.

La crispation de DPAG sur ces licences s'explique aisément, les services offerts par la concurrence étant proches des prestations liées aux obligations de service universel ; elles menacent donc directement ses activités sous monopole. Et la ligne de défense de DPAG porte sur un point particulièrement sensible : l'acheminement du courrier le jour même ou durant la nuit. En prenant livraison du courrier après 17 heures pour le distribuer le lendemain avant midi, ses concurrents assurent une qualité de service effectivement supérieure à celle que doit garantir DPAG au titre des obligations de service universel : une durée d'acheminement d'au moins $80 \%$ à $\mathrm{J}+1$ et de $95 \%$ à $\mathrm{J}+2$. Selon le contrôle qualité effectué régulièrement par la RegTP, DPAG frise en 2003 respectivement 88 et $99 \%$. C'est donc sur la différence entre ces deux derniers taux, d'une part, et, d'autre part, le $100 \%$ garanti par la concurrence que porte le litige. L'enjeu central est donc la délimitation du périmètre de la licence légale exclusive.

Depuis 1999, les tribunaux administratifs régionaux donnent alternativement raison à DPAG ou tort aux concurrents ; la plupart des affaires a été renvoyée de-
Des procès systématiques contre les détenteurs d'une licence $D$

Licences D versus licence légale exclusive

A la jurisprudence de délimiter le monopole 
vant la Cour fédérale de Cassation qui devrait, selon toutes prévisions, statuer courant 2004. Son arrêt permettra enfin de clarifier la situation. La RegTP espère une jurisprudence favorable à une concurrence fair play. Dans deux récents arrêts (août et octobre 2003), la Cour avait en effet, dans d'autres litiges opposant DPAG et ses concurrents et la RegTP, adopté des positions en ce sens. En tout état de cause, son arrêt permettra de définir enfin ce qui relève du monopole temporaire de DPAG ou non.

L'ÉTAT DU MARCHÉ ALLEMAND DE LA LETTRE EST PARADOXAL. Alors que la réglementation favorise l'émergence d'un secteur concurrentiel, l'opérateur historique se crispe sur son monopole, juridiquement résiduel, mais de facto amplement préservé. DPAG défend sa licence légale exclusive en arguant de ses obligations de service universel, saisissant systématiquement les tribunaux pour évincer ses concurrents émergents. Le principal obstacle à la genèse d'un marché des services postaux réside dans un haut risque procédurier aboutissant à une distorsion de concurrence.

Ce risque est le fait des imprécisions d'une réglementation adoptée à une époque où la préoccupation première du législateur concernait le bon déroulement de la privatisation de Deutsche Post AG et l'aménagement d'une transition progressive vers l'ouverture totale du marché. Cette phase, puisqu'elle était initialement limitée à 5 ans, légitimait alors une entorse - à titre provisoire exclusivement - au droit commun de la concurrence, soumettant à une régulation dérogatoire, spécifique, un marché émergent. Une autorité ad hoc avait été instituée, chargée de veiller au strict respect de la libre concurrence par les opérateurs anciennement en situation de monopole (poste et télécommunications). Mais cette RegTP, elle-même provisoire, n'a pas les pouvoirs de l'Office fédéral des Cartels : le gouvernement fédéral peut casser ses décisions, ainsi qu'il l'a fait en 2000 à propos de la tarification de la lettre standard. La RegTP, chargée par la loi de favoriser la constitution du marché, se révèle dès lors d'autant plus impuissante, dans la réalité, à remplir ces missions, que le monopole de Deutsche Post AG a été récemment prolongé de six ans.

Le problème de fond que révèle la difficile émergence d'un marché de la lettre tient au conflit entre droit commun de la concurrence et régulation sectorielle. II révèle la difficulté d'appréhender le marché des services avec les règles et les instances datant de l'ère industrielle. Le gouvernement fédéral a cherché à le résoudre en recourant à une forme d'autorégulation tripartite sous contrôle rapproché de l'Etat plutôt qu'en étendant les compétences de l'Office fédéral des Cartels aux secteurs anciennement en situation de " monopole naturel ». Aujourd'hui, il est question d'étendre les compétences de la RegTP au marché de l'énergie. De provisoire, l'instance de régulation deviendrait alors pérenne. Le retard pris sur le calendrier initial de la libéralisation du marché de la lettre aura contribué à favoriser cette tendance. Reste la question des pouvoirs du nouveau gardien de la concurrence. Elle est en suspens. Pour l'instant, c'est à la Cour fédérale de Cassation de les exercer via sa jurisprudence à venir.

\section{Indications bibliographiques}

BOURGEOIS Isabelle, «La libéralisation du marché postal allemand : entre service public et marché », in Bourgeols I. (dir), Allemagne 2001, Regards sur une économie en mutation, CIRAC, 2001

BUNDESVERBAND INTERNATIONALER EXPRESS- UND KURIERDIENSTE - BIEK (www.biek.de) BUNDESVERFASSUNGSGERICHT, Beschluss vom 7. Oktober 2003 - 1 BvR 1712/01 (site: www. bundesverfassungsgericht.de)

Monopolkommission, Sondergutachten. Telekommunikation und Post 2003 : Wettbewerbsintensivierung in der Telekommunikation - Zementierung des Postmonopols, Bonn, 11-12-2003 (site: www.monopolkommission.de)

Regulierungsbehörde für POSt UND TeleKommuniKation - RegTP, Tätigkeitsbericht 2002/2003, Bonn, décembre 2003 (site: www.regtp.de) 